\title{
FDFD simulation for high resolution eddy-current testing method
}

\author{
Takuya SAITO $^{1}$, Hitoshi MIKADA ${ }^{1}$, Tadanori GOTO ${ }^{1}$ and Junichi TAKEKAWA ${ }^{1}$ \\ ${ }^{1}$ Dept. of Civil and Earth Res. Eng., Kyoto University
}

\begin{abstract}
The applicability and the feasibility of eddy-current testing method for the detection of wall thinning and surface crack of steel structure have been practically confirmed by field and laboratory experiments. Qualitative analysis of cracks has been empirically understood by this method. There has, however, been a growing demand to quantitatively evaluate the cracks and we are inspired to use time series of induced magnetic field induced in the vicinity of cracks. For this objective, we first developed a numerical simulator for the high accuracy eddy-current method. As a result, the effect of surface cracks of a steel plate to magnetic field was confirmed as follows: the induced magnetic field intensity increases near the edge of cracks and decays above the cracks with the distance to the edge. The deeper and wider cracks become, the more the magnetic field intensity is attenuated. Due to the limitation of our simulation schemes, the response of magnetic field intensity whose detectable scale of cracks was no smaller than mm order in $2 \mathrm{D}$ simulation. We are introducing a method that could allow us to confirm much finer detectability.
\end{abstract}

\section{INTRODUCTION}

Eddy-current testing method is applied for NDE (Non-destructive evaluation), such as detection of metal cracks. Several examinations show that the parameters of the crack depth affect the detection signal (Gui Yun Tian, 2005). This difference in the induced electromotive force is used to detect cracks. Because this technique is sensitive to material constants, it is difficult to detect cracks or scratches present on the backside of the material, and it did not result in a quantitative evaluation. Especially, it is difficult to obtain the information for the depth of the scratches and cracks. It is possible to determine the presence of cracks in the inspection technique of today, but to determine the state of the wound is difficult. So, in order to grasp accurately the shape of and the progress of the metal cracks, it is necessary to inspect with high accuracy. In general, in order to raise the resolution to be obtained in the electromagnetic induction method, it is necessary to set high frequency electromagnetic field excitation. However, since the electromagnetic field of high frequency is highly attenuated, only the cracks around the metal surface could be detected. This indicates that the method to investigate the cracks hidden inside the metal with high accuracy have to be established.

We conduct numerical simulations to evaluate the applicability and feasibility of the quantitative evaluation of crack depth. In order to calculate the induced magnetic field due to the cracks, a 2.5 dimensional finite-difference frequency domain technique is used to solve Maxwell's equations numerically. In this technique, the two-dimensional structure and the three-dimensional electromagnetic field are assumed. As the model, a two-layer structure consisting of seawater and steel plate containing cracks is assumed. To estimate characteristic of the induced magnetic field, we simulate for a variety of models with different depth and width of the cracks, and investigate the effect of them on the observed data. The magnetic field data derived from 2.5D-FDFD is wave number domain. Through the inverse Fast Fourier Transform of the results obtained by $2.5 \mathrm{D}-\mathrm{FDFD}$, the magnetic field in space domain and time domain with phase information is calculated. This information helps us identify the position of the crack by using the phase information due to crack.

\section{THEORY AND METHOD}

\section{(1) Maxwell's equation}

In this study, we excite a magnetic field on the surface of the iron, and calculate the secondary magnetic field. To calculate the magnetic field, the following equations derived from Maxwell's equation are used as:

$$
\nabla \times \nabla \times \mathbf{H}+i \omega \mu \hat{\sigma} \mathbf{H}=-\hat{\sigma} \mathbf{J}_{m}^{s}
$$




$$
\hat{\sigma}=\sigma+i \omega \varepsilon
$$

where $\mathbf{H}[\mathrm{A} / \mathrm{m}]$ is magnetic field intensity, i is imaginary unit, $\omega$ is angular frequency, $\mu[\mathrm{H} / \mathrm{m}]$ is permeability, $\sigma[\mathrm{S} / \mathrm{m}]$ is conductivity, $\varepsilon[\mathrm{F} / \mathrm{m}]$ is permittivity, $\mathbf{J}_{m}^{s}[\mathrm{~A} / \mathrm{m}]$ is a magnetic source.

\section{(2) 2.5D-FDFD method}

Three-dimensional Maxwell equations are discretized by a 4th-order finite-difference scheme (Haber et al., 2001). Because of the huge matrix, it is very time consuming and needs large computational memory to solve it. Therefore, 2.5D-FDFD method was devised (Mitsuhata, 2000). In this method, two-dimensional structure and three-dimensional electromagnetic field are assumed. The procedure to calculate magnetic field using 2.5D-FDFD method is shown in Figure1.

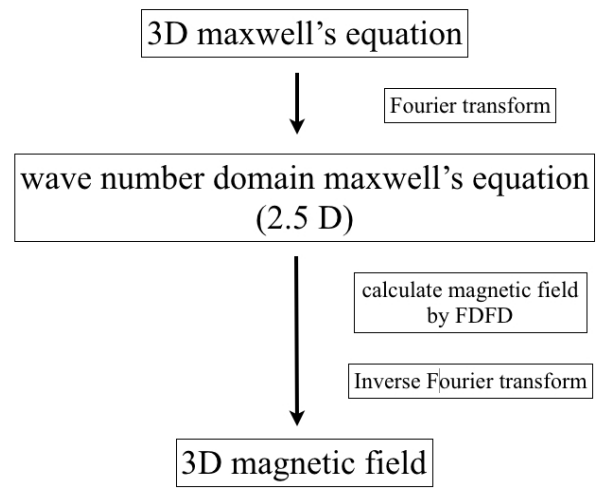

Figure 1 The procedure to calculate 2.5D-FDFD method.

\section{(3) Wave number domain maxwell's equation}

In 2.5D-FDFD method, the electromagnetic field along the $\mathrm{y}$ direction is transformed to wave number domain as below:

$$
\tilde{\mathbf{H}}\left(x, k_{y}, z\right)=\frac{1}{\pi} \int_{0}^{\infty} \mathbf{H}(x, y, z, \omega) d k_{y}
$$

where $\tilde{\mathbf{H}}$ means magnetic field in wave number domain, and $\mathrm{k}_{\mathrm{y}}$ means wave number in y-direction. Using this equation, the following equations can be derived.

$$
\begin{array}{r}
\left(k_{y}^{2}+i \omega \mu \hat{\sigma}-\frac{\partial^{2}}{\partial z^{2}}\right) \tilde{\mathbf{H}}_{x}+i k_{y} \frac{\partial}{\partial x} \tilde{\mathbf{H}}_{y}+\frac{\partial^{2}}{\partial x \partial z} \tilde{\mathbf{H}}_{z}=-\hat{\sigma} \mathbf{J}_{x m}^{s}(4) \\
i k_{y} \frac{\partial}{\partial x} \tilde{\mathbf{H}}_{x}-\left(\frac{\partial^{2}}{\partial x^{2}}+\frac{\partial^{2}}{\partial z^{2}}-i \omega \mu \hat{\sigma}\right) \tilde{\mathbf{H}}_{y}+i k_{y} \frac{\partial}{\partial z} \tilde{\mathbf{H}}_{z}=-\hat{\sigma} \mathbf{J}_{y m}^{s}(5) \\
\frac{\partial^{2}}{\partial x \partial z} \tilde{\mathbf{H}}_{x}+i k_{y} \frac{\partial}{\partial z} \tilde{\mathbf{H}}_{y}+\left(k_{y}^{2}+i \omega \mu \hat{\sigma}-\frac{\partial^{2}}{\partial x^{2}}\right) \tilde{\mathbf{H}}_{z}=-\hat{\sigma} \mathbf{J}_{z m}^{s}(6)
\end{array}
$$

The simultaneous equation (4), (5) and (6) become as follows:

$$
\tilde{\mathbf{A}} \tilde{\mathbf{x}}=\tilde{\mathbf{b}}
$$

where $\tilde{\mathbf{A}}$ is coefficient matrix, $\tilde{\mathbf{x}}$ is magnetic field, $\tilde{\mathbf{b}}$ is magnetic source. Equation (7) was solved using iterative method (Van Der Vorst, 1992).

\section{(4) Inverse fast fourier transform}

After solving the equation (7), the electromagnetic fields in the $\mathrm{k}_{\mathrm{y}}$-domain are transformed to the space domain and time domain by using following inverse Fourier transformations. They are evaluated by

$$
\begin{aligned}
\mathbf{H}(x, y, z, \omega) & =\frac{1}{\pi} \int_{0}^{\infty} \tilde{\mathbf{H}}\left(x, k_{y}, z, \omega\right) d k_{y} \\
\mathbf{H}(x, y, z, t) & =\frac{1}{\pi} \int_{0}^{\infty} \mathbf{H}(x, y, z, \omega) d \omega
\end{aligned}
$$

In inverse Fast Fourier Transform (IFFT), these inverse Fourier transforms (8) and (9) were used. For IFFT, 128 wave numbers $(\sim 12.5)$ and frequencies $(\sim 4 \mathrm{kHz})$ were used.

\section{RESULTS AND DISCUSSIONS}

In this section, the effect of the crack on the observed magnetic field is examined. The crack detection of sheet pile in the coast was assumed. So two-layer structure of seawater and conducting material, iron was used as simulation models (Figure 2).

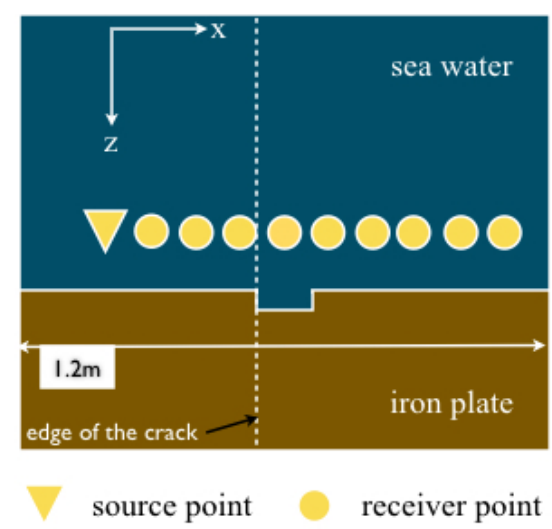

Figure 2 Simulation model (a). Triangle and circles represent source and receivers (150points), respectively. The interval of receivers is $0.08 \mathrm{~m}$. 
Figure 3 shows the induced magnetic field intensity in the space domain in the case of model (a). The feature could be observed that the induced magnetic field intensity increases near the edge of the cracks and decays above the cracks could be observed.

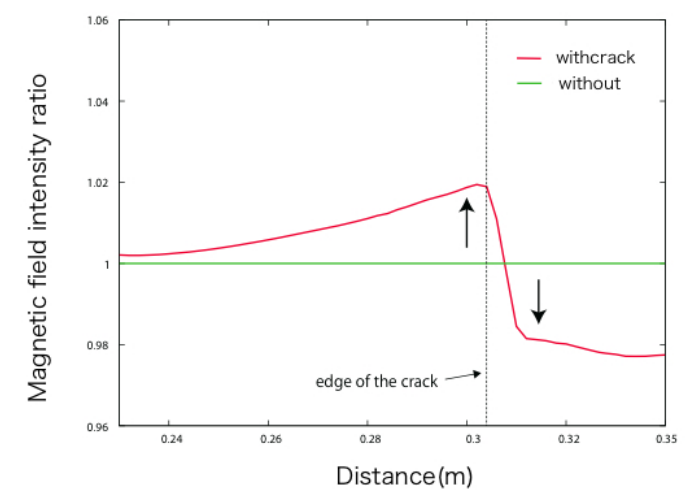

Figure 3 The induced magnetic field intensity in the case of model (a). X-axis is the distance from the source point. Red line with crack was taken by the ratio of Green line without crack.

We also calculated the induced magnetic field in time domain by inverse fast Fourier transform in frequency domain. We used $2 \mathrm{kHz}$ for Nyquist frequency. Interval is $31.25 \mathrm{~Hz}$ and the number of sampling data is 128 .

Figure 4 shows the envelope of the induced magnetic field (vertical direction) in time domain with and without crack. The figures are z-direction magnetic field received with different offset distances. The difference of their amplitude and phase could be seen from the comparison between the Red line with crack and Green line without crack. When steel plate has the crack, the magnetic field is smaller and delayed more than the case without crack.
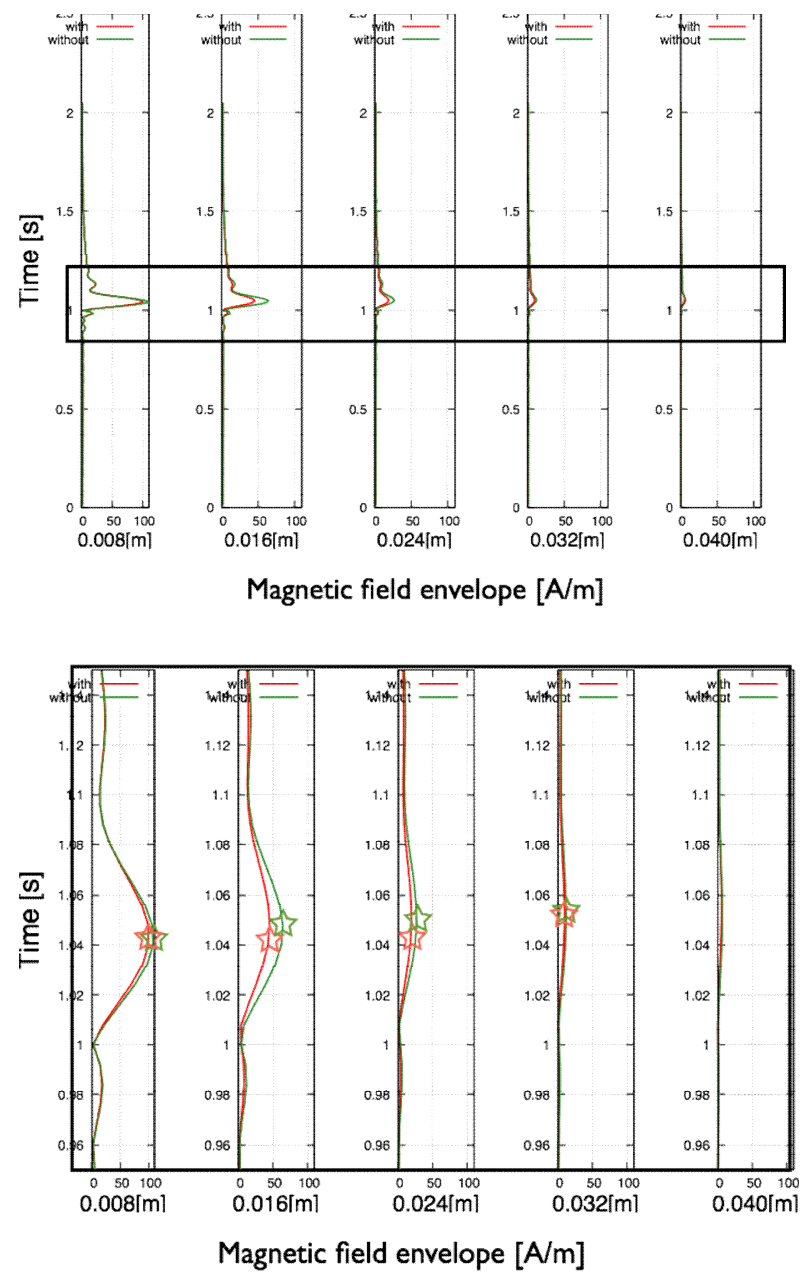

Figure 4 These figures are the envelope of the magnetic field in time domain. Distance from the transmitting source is away from left to right. Red line shows with crack and green line indicates without crack.

\section{CONCLUSIONS}

We conducted numerical simulations of magnetic field for investigating the applicability and feasibility of eddy-current testing method to improve the accuracy of the detection capability. The observed magnetic field is affected by the irregularity of objective materials such as crack. From the results in time-space domain, there is the tendency that the induced magnetic field intensity increases near the edge of the crack and decays above the crack. The larger the crack length and width are, the more attenuated the magnetic field intensity becomes. Numerical simulation results indicated that the response of magnetic field intensity could be calculated whose detectable scale of cracks is no smaller than mm order. From the results in time domain, the difference of phase and 
amplitude of magnetic field due to crack on the metal plate could be confirmed.

This information contains the position of crack, so we would like to estimate the position of the crack from the phase information for future work.

\section{REFERENCES}

1) E. Haber, U. Ascher, 2001, Fast Finite Volume Simulation of 3D Electromagnetic problems with Highly Discontinuous Coefficients, SIAM Journal on Scientific Computing, Vol. 22, 1943-1961

2) Gui Yun Tian, 2005, Reduction of lift-off effects for pulsed eddy current NDT, NDT\&E International, 38, 319-3224

3) H. A. Van Der Vorst, 1992, BI-CGSTAB : A first and smoothly converging variant of BI-CG for the solution of nonsymmetric linear systems, SIAM Journal on Scientific Computing, Vol.13, No. 2, 631-644

4) Yuji Mitsuhata, 2000, 2-D electromagnetic modeling by finite-element method with a dipole source and topography, Geophysics, 65, 465-475 